\title{
Ein Beitrag zu den Diophantischen Approximationen reeller Zahlen
}

\author{
Robert Baggenstos
}

Robert Baggenstos studierte Mathematik und Astronomie an der Universität Bern, arbeitete danach in der Lehrerausbildung und unterrichtet heute Mathematik und Astronomie an der Kantonsschule in Solothurn.

\section{Einleitung}

Der Versuch, reelle Zahlen mit rationalen zu approximieren, bildet ein klassisches Thema der Zahlentheorie. Von MinkowsKi [4] wurde dieses Gebiet mit „Diophantische Approximationen“" umschrieben, und dieser Begriff wird heute in der Regel dann verwendet, wenn es darum geht, bei gegebenem $r \in \mathbb{R}$ den Approximationsfehler $\left|r-\frac{A}{B}\right|$ durch geeignete Wahl von ganzen Zahlen $B>0$ und $A$ nach oben abzuschätzen, oder aber ihn möglichst klein zu gestalten.

Auf der Suche nach gültigen Aussagen in diesem Problemfeld stösst man in der Regel auf Sätze, welche in drei Klassen unterteilt werden können:

I. Existenzsätze für den Fall, dass der Wert des Nenners $B \in \mathbb{N}$ nicht nach oben beschränkt ist. Diese Sätze besagen dann etwa, dass unendlich viele (gekürzte) Brüche $\frac{A}{B}$ existieren, so dass gilt $\left|r-\frac{A}{B}\right|<c(B)$, wo $c(B)$ eine vom Nenner $B$ abhängige obere Schranke bildet.

Bei dieser Klasse von Aussagen sprechen wir im Folgenden von „Approximationen mit beliebig grossen Nennern“.

Ausgangslage zu den nachfolgenden Untersuchungen bildet ein sehr praktisches Problem: Bei der Konstruktion eines mechanischen Werkes für eine Uhr mit ,astronomischen Indikatoren“ (Mondphasen, siderischer Tag, tropisches Jahr, u.a.) ist mit Hilfe mehrerer Zahnräder (selbstverständlich mit beschränkt grossen und kleinen Zähnezahlen) ein bestimmtes Drehverhältnis $r$ zwischen zwei Achsen möglichst genau zu erzielen. Dabei stösst man auf das Grundproblem der Diophantischen Approximation, nämlich die Grösse $\left|r-\frac{\alpha_{1} \cdot \alpha_{2} \cdot \ldots \cdot \alpha_{n}}{\beta_{1} \cdot \beta_{2} \cdot \ldots \cdot \beta_{n}}\right|$ möglichst klein zu halten, wobei $\alpha_{i}$ und $\beta_{i}$ natürliche Zahlen mit $m \leq \alpha_{i}, \beta_{i} \leq M(i=1, \ldots, n)$ sind. 
II. Existenzsätze für den Fall, dass der Wert des Nenners $B \in \mathbb{N}$ nach oben beschränkt ist. Diese Sätze besagen dann etwa, dass mindestens ein Bruch $\frac{A}{B}$ existiert, so dass gilt $\left|r-\frac{A}{B}\right|<c(B)$, wo $c(B)$ wiederum eine vom Nenner B abhängige obere Schranke bildet.

Bei dieser Klasse von Aussagen sprechen wir im Folgenden von „Approximationen mit beschränkt grossen Nennern“".

III. Algorithmische Wege zur Bestimmung der bestmöglichen Approximation einer reellen Zahl $r \in \mathbb{R}$ mit einem gekürzten Bruch $\frac{A}{B}$, wo der natürliche Nenner $B$ (und eventuell auch der ganzzahlige Zähler $A$ ) aus einer bestimmten endlichen Teilmenge von $\mathbb{N}$ (resp. von $\mathbb{Z}$ ) stammen muss.

Bei dieser Klasse von Aussagen sprechen wir im Folgenden von „Approximationsalgorithmen".

$\mathrm{Zu}$ jeder der drei Klassen sollen hier einige der bereits bekannten Erkenntnisse zusammengestellt werden, wobei Vollständigkeit weder angestrebt noch möglich ist. Ferner sollen dazu auch einige zusätzliche Aussagen hergeleitet werden.

\section{Grundlagen}

Wo im Folgenden mit $\frac{A}{B}$ eine rationale Zahl erscheint, seien $A$ und $B$ stets ganze Zahlen und $B>0$. Die zu approximierende Grösse sei ohne weitere Hinweise eine beliebige reelle Zahl, und diese wird in der Regel mit $r$ bezeichnet.

Es geht nun also darum, die Differenz zwischen einer reellen Zahl $r$ und einer rationalen Zahl $\frac{A}{B}$ nach oben abzuschätzen mit einer positiven Schranke $c(B)$ :

$$
\left|r-\frac{A}{B}\right|<c(B) \quad \text { oder allenfalls } \quad\left|r-\frac{A}{B}\right| \leq c(B) .
$$

Selbstverständlich weisen zwei benachbarte Bruchzahlen $\frac{A}{B}$ und $\frac{A+1}{B}$ mit gleichem Nenner $B$ eine Differenz von $\frac{1}{B}$ auf, weshalb für jede reelle Zahl $r$ und für jeden natürlichen Nenner $B$ (sowie eine geeignete ganze Zahl $A$ ) gilt:

$$
\left|r-\frac{A}{B}\right| \leq \frac{1}{2 B}
$$

Und weil dabei die Gleichheit höchstens dann eintreten kann, wenn $r$ rational ist, gilt für jedes $r \in \mathbb{R} \backslash \mathbb{Q}$ und für jeden natürlichen Nenner $B$ (sowie eine geeignete ganze Zahl $A$ ) sogar:

$$
\left|r-\frac{A}{B}\right|<\frac{1}{2 B}
$$

Für die Kettenbruch-Darstellung von irrationalen Zahlen verwenden wir anstelle der Form

$$
q_{0}+\frac{1}{q_{1}+\frac{1}{q_{2}+\frac{1}{q_{3}+\ldots}}}
$$


die einfachere Darstellung $\left[q_{0} ; q_{1}, q_{2}, q_{3}, \ldots\right]$. Dabei ist $q_{0} \in \mathbb{Z}$ und $q_{i} \in \mathbb{N}$ für alle $i \geq 1$. Für rationale Zahlen verwenden wir dann sinngemäss die Form $\left[q_{0} ; q_{1}, q_{2}, \ldots, q_{n}\right]$. Dabei ist wiederum $q_{0} \in \mathbb{Z}$ und $q_{i} \in \mathbb{N}$ für alle $i \geq 1$ sowie $q_{n} \geq 2$, womit auch bei rationalen Zahlen die Eindeutigkeit ihrer Kettenbruchdarstellung gewährleistet wird.

\section{Approximationen mit beliebig grossen Nennern}

In diesem Abschnitt betrachten wir Diophantische Approximationen von reellen Zahlen $r$, wobei der Nenner $B$ der Bruchzahlen $\frac{A}{B}$ aus einer bestimmten, aber jedenfalls unendlich mächtigen Teilmenge $\mathbb{G}$ der natürlichen Zahlen gewählt werden kann.

Zunächst betrachten wir hier ein paar Aussagen zur Approximation rationaler Zahlen. Grundsätzlich muss man zunächst feststellen, dass sich rationale Zahlen weniger gut approximieren lassen als irrationale, denn für zwei voneinander verschiedene rationale Zahlen $\frac{n}{m}$ und $\frac{A}{B}$ gilt stets (wenn diese beiden Brüche vollständig gekürzt sind):

$$
\left|\frac{n}{m}-\frac{A}{B}\right| \geq \frac{1}{m B} .
$$

Geht es also beispielsweise darum, eine ganze Zahl zu approximieren $(m=1)$ mit einer von dieser ganzen Zahl verschiedenen rationalen Zahl $\frac{A}{B}$, dann kann dabei der Fehler selbstverständlich nicht kleiner sein als $\frac{1}{B}$. Und falls man eine rationale Zahl aus der Menge $\{ \pm 0.5, \pm 1.5, \pm 2.5, \ldots\}$ (also mit $m=2$ ) mit einer von ihr verschiedenen rationalen Zahl $\frac{A}{B}$ approximieren will, dann beträgt nach (3) der Approximationsfehler mindestens $\frac{1}{2 B}$. Für die übrigen rationalen Zahlen lässt sich hingegen die Aussage (1) leicht verschärfen:

Satz 1 Zu jeder rationalen Zahl $r \in \mathbb{Q} \backslash\{0, \pm 0.5, \pm 1, \pm 1.5, \pm 2, \ldots\}$ existieren unendlich viele (gekürzte) Bruchzahlen $\frac{A}{B}$ mit der Eigenschaft

$$
\left|r-\frac{A}{B}\right|<\frac{1}{2 B} .
$$

Beweis. Sei etwa $r=\frac{n}{m}$ (mit $n \in \mathbb{Z}$ und mit $m \in \mathbb{N}$ und $m>2$ sowie $(n, m)=1$ ). Die Gleichheit in (1) tritt genau dann ein, wenn $A \in \mathbb{Z}$ und $B \in \mathbb{N}$ existieren, so dass $r$ exakt in der Mitte zwischen $\frac{A}{B}$ und $\frac{A+1}{B}$ liegt. Daraus ergibt sich:

$$
\frac{n}{m}=\frac{1}{2} \cdot\left(\frac{A}{B}+\frac{A+1}{B}\right)=\frac{2 A+1}{2 B} .
$$

Sei nun etwa $B$ eine Primzahl und $B \neq \frac{m}{2}$. Dann kann der in (4) rechts stehende Bruch höchstens mit $B$ gekürzt werden (da sein Zähler ungerade ist).

1. Falls dieser Bruch nicht kürzbar ist, gilt $m=2 B$, was im Widerspruch steht zu $B \neq \frac{m}{2}$.

2. Falls dieser Bruch mit $B$ kürzbar ist, gilt $m=2$, was im Widerspruch steht zu $m>2$. 
Somit kann die Gleichheit in (1) mindestens für alle $B$, welche Primzahlen und von $\frac{m}{2}$ verschieden sind, nicht eintreten, woraus bereits Satz 1 folgt.

Satz 1 lässt sich noch wesentlich verallgemeinern:

Satz 2 Es sei $r=\frac{n}{m}$ eine rationale Zahl mit $m \in \mathbb{N}$ und $(n, m)=1$. Ferner sei $m>k \in \mathbb{N}$ beliebig. Dann existieren unendlich viele (gekürzte) Bruchzahlen $\frac{A}{B}$ mit der Eigenschaft

$$
\left|\frac{n}{m}-\frac{A}{B}\right|<\frac{1}{k B}
$$

Beweis. Bekanntlich besitzt die Diophantische Gleichung

$$
B n-A m=1
$$

für $n, m \in \mathbb{Z}$ und $(n, m)=1$ stets ganzzahlige Lösungen für $A$ und $B$. Sei $A_{0}, B_{0}$ eine dieser Lösungen. Also gilt dann $B_{0} n-A_{0} m=1$. Dann sind auch alle

$$
\begin{aligned}
& B(t)=B_{0}+m t \\
& A(t)=A_{0}+n t
\end{aligned}
$$

(mit $t \in \mathbb{Z}$ ) ganzzahlige Lösungen von (5). Für alle hinreichend grossen Werte von $t$ gilt wegen $m>0$ auch $B(t)=B_{0}+m t>0$. Da die Werte $A(t)$ und $B(t)$ die Gleichung (5) erfüllen, sind sie zudem sicher teilerfremd. Somit existieren unendlich viele teilerfremde $A \in \mathbb{Z}$ und $B \in \mathbb{N}$, für welche gilt $B n-A m=1$. Daraus folgt durch Division durch $B$ und durch $m$, und wegen $m>k$ :

$$
0<\frac{n}{m}-\frac{A}{B}=\frac{1}{m B}<\frac{1}{k B}
$$

also etwas mehr noch als Satz 2 behauptet.

Nun betrachten wir ein paar Aussagen zur Approximation irrationaler Zahlen.

Satz 3 Zu jeder irrationalen Zahl $r \in \mathbb{R} \backslash \mathbb{Q}$ existieren unendlich viele (gekürzte) Bruchzahlen $\frac{A}{B}$ mit

$$
\left|r-\frac{A}{B}\right|<\frac{1}{\sqrt{5} \cdot B^{2}} .
$$

Bemerkung. Dieser Satz wurde 1889 von A. HURWITZ zunächst mit Hilfe der Theorie der Kettenbrüche hergeleitet. Später wurden verschiedene andere Beweise gefunden, beispielsweise von A.J. KHINTCHINe [2]. Ausserdem hat HURWITZ nachgewiesen, dass man diese Abschätzung nicht weiter verschärfen kann, ohne für die zu approximierende Irrationalzahl $r$ einschränkende Voraussetzungen einzuführen. So lässt sich zeigen, dass für die Zahl $r=\frac{\sqrt{5}-1}{2}$ (Goldener Schnitt!) nur endlich viele gekürzte $\frac{A}{B}$ existieren, so dass gilt $\left|r-\frac{A}{B}\right|<\frac{1}{c \cdot B^{2}}$, sobald $c>\sqrt{5}$ ist. 
Satz 4 Zu jeder irrationalen Zahl $r \in \mathbb{R} \backslash \mathbb{Q}$ existieren von jedem einzelnen der drei Typen $\frac{\text { ungerade }}{\text { ungerade }}, \frac{\text { gerade }}{\text { ungerade }}, \frac{\text { ungerade }}{\text { gerade }}$ unendlich viele (gekürzte) Bruchzahlen $\frac{A}{B}$ mit

$$
\left|r-\frac{A}{B}\right|<\frac{1}{B^{2}}
$$

Satz 5 Zu jeder irrationalen Zahl $r \in \mathbb{R} \backslash \mathbb{Q}$ existieren von mindestens zwei der drei Typen $\frac{\text { ungerade }}{\text { ungerade }}, \frac{\text { gerade }}{\text { ungerade }}$, $\frac{\text { ungerade }}{\text { gerade }}$ unendlich viele (gekürzte) Bruchzahlen $\frac{A}{B}$ mit

$$
\left|r-\frac{A}{B}\right|<\frac{1}{2 B^{2}} .
$$

Bemerkung. Die Sätze 4 und 5 wurden 1940 von R.M. Robinson [7] mit Hilfe der Theorie der Kettenbrüche hergeleitet. W.T. ScOTT [8] leitete Satz 4 ebenfalls 1940 unter Zuhilfenahme geometrischer Transformationen her.

\section{Approximationen mit beschränkt grossen Nennern}

In diesem Abschnitt betrachten wir Diophantische Approximationen von reellen Zahlen $r$, wenn der Nenner $B$ der Bruchzahlen $\frac{A}{B}$ aus einer bestimmten endlichen Teilmenge $\mathbb{G}$ der natürlichen Zahlen gewählt werden kann.

Satz 6 Zu jeder reellen Zahl $r \in \mathbb{R}$ und zu jeder natürlichen Schranke $M \in \mathbb{N}$ existiert mindestens eine Bruchzahl $\frac{A}{B}$ mit $B \leq M$ und mit der Eigenschaft

$$
\left|r-\frac{A}{B}\right|<\frac{1}{M B} \leq \frac{1}{B^{2}} .
$$

Wir beweisen hier gleich eine etwas allgemeinere Aussage:

Satz 7 Zu jeder reellen Zahl $r \in \mathbb{R}$ und zu den beiden beliebigen natürlichen Schranken $n, M \in \mathbb{N}$ (mit $n \leq M$ ) existiert mindestens eine Bruchzahl $\frac{A}{B}$ mit $n \leq B \leq M$ und mit der Eigenschaft

$$
\left|r-\frac{A}{B}\right|<\frac{1}{\left[\frac{M}{n}\right] B} .
$$

Dabei ist $[x]=\max \{p \mid p \in \mathbb{Z} \wedge p \leq x\}$.

Beweis. Es sei $z_{t}:=t r-[t r]$, mit $t=0,1,2, \ldots, M$. Diese $M+1$ Zahlen liegen alle im Intervall $[0,1)$. Es sei nun $c:=\left[\frac{M}{n}\right]$. Nun unterteilen wir das Einheitsintervall $[0,1)$ in $c$ gleich grosse Teilintervalle, gemäss $[0,1)=\left[0, \frac{1}{c}\right) \cup\left[\frac{1}{c}, \frac{2}{c}\right) \cup \ldots \cup\left[\frac{c-1}{c}, 1\right)$. Von den Zahlen $z_{t}$ liegen in einem solchen Teilintervall im Durchschnitt

$$
\frac{M+1}{c}=\frac{M+1}{\left[\frac{M}{n}\right]} \geq \frac{M+1}{\frac{M}{n}}>n
$$

Zahlen. Also existiert mindestens ein solches Teilintervall, welches mindestens $n+1$ dieser Zahlen $z_{t}$ enthält, etwa die Zahlen mit den Indizes $t_{1}, t_{2}, \ldots, t_{n+1}$ und mit $t_{1}<t_{2}<\ldots<$ 
$t_{n+1}$. Dann gilt mit Sicherheit $n \leq t_{n+1}-t_{1} \leq M$. Da die entsprechenden Zahlen $z_{t}$ im selben Teilintervall liegen, gilt somit

$$
\left|z_{t_{n+1}}-z_{t_{1}}\right|<\frac{1}{c}
$$

also: $\left|t_{n+1} r-\left[t_{n+1} r\right]-\left(t_{1} r-\left[t_{1} r\right]\right)\right|=\left|\left(t_{n+1}-t_{1}\right) r-\left(\left[t_{n+1} r\right]-\left[t_{1} r\right]\right)\right|<\frac{1}{c}$. Mit $A=\left[t_{n+1} r\right]-\left[t_{1} r\right]$ und mit $B=t_{n+1}-t_{1}$ gilt: $A \in \mathbb{Z}, B \in \mathbb{N}, n \leq B \leq M$, sowie $|B r-A|<\frac{1}{c}$. Nach Division durch $B$ ergibt sich unmittelbar die Aussage von Satz 7 .

Bemerkungen. Satz 6 wurde 1842 von G.P. LEJEUne DiRICHLET [3] mit Hilfe des Schubfachprinzips hergeleitet, welches hier im Beweis des Satzes 7 verwendet wurde. Satz 6 ergibt sich natürlich unmittelbar aus Satz 7 für $n=1$. Ebenso ergeben sich die Sätze 6 und 7 als Spezialfälle aus dem Satz 9. Auf die Voraussetzung $M \in \mathbb{N}$ (und $n \in \mathbb{N}$ ) kann nach geringfügiger Modifikation der Beweisführung im letzten Satz auch verzichtet werden.

Satz 8 Es existieren höchstens zwei verschiedene (gekürzte) Bruchzahlen $\frac{A}{B}$, welche die Bedingungen von Satz 6 erfüllen.

Beweis. Es sei $r \in \mathbb{R}$ und $M \in \mathbb{N}$ beliebig. Innerhalb der Farey-Folge der Ordnung $M^{1)}$ betrachten wir die beiden konsekutiven Bruchzahlen $\frac{a_{i}}{b_{i}}$ und $\frac{a_{i+1}}{b_{i+1}}$, für welche gilt: $\frac{a_{i}}{b_{i}} \leq$ $r<\frac{a_{i+1}}{b_{i+1}}$. Innerhalb einer solchen Farey-Folge sind bekanntlich alle Nenner $b_{j} \leq M$, und für zwei konsekutive Brüche der Folge gilt zudem $\frac{a_{i+1}}{b_{i+1}}-\frac{a_{i}}{b_{i}}=\frac{1}{b_{i} b_{i+1}}$. Somit gilt $\frac{a_{i+1}}{b_{i+1}}-\frac{a_{i}}{b_{i}} \geq \frac{1}{b_{i} M}$ und $\frac{a_{i+1}}{b_{i+1}}-\frac{a_{i}}{b_{i}} \geq \frac{1}{b_{i+1} M}$. Für einen beliebigen Bruch $\frac{a_{i-k}}{b_{i-k}}$ dieser Folge, welcher unterhalb von $\frac{a_{i}}{b_{i}}$ liegt, gilt daher:

wegen

$$
r-\frac{a_{i-k}}{b_{i-k}} \geq \frac{1}{b_{i} b_{i-1}}+\frac{1}{b_{i-1} b_{i-2}}+\cdots+\frac{1}{b_{i-k+1} b_{i-k}},
$$

und somit

$$
r-\frac{a_{i-k}}{b_{i-k}} \geq \frac{1}{M b_{i-1}}+\frac{1}{M b_{i-2}}+\cdots+\frac{1}{M b_{i-k}},
$$

$$
r-\frac{a_{i-k}}{b_{i-k}} \geq \frac{1}{M}\left(\frac{1}{b_{i-1}}+\frac{1}{b_{i-2}}+\cdots+\frac{1}{b_{i-k}}\right) \geq \frac{1}{M b_{i-k}} .
$$

Ebenso kann man zeigen, dass für jeden beliebigen Bruch $\frac{a_{i+1+k}}{b_{i+1+k}}$ oberhalb von $\frac{a_{i+1}}{b_{i+1}}$ gilt:

$$
\frac{a_{i+1+k}}{b_{i+1+k}}-r>\frac{1}{M b_{i+1+k}} .
$$

Somit verbleiben lediglich die beiden konsekutiven Brüche $\frac{a_{i}}{b_{i}}$ und $\frac{a_{i+1}}{b_{i+1}}$, welche die Bedingungen von Satz 7 allenfalls zu erfüllen vermögen.

Bemerkung. Der Satz 8 wurde bereits von SIERPINSKI [9] nachgewiesen.

\footnotetext{
${ }^{1)}$ Ordnet man die gekürzten Brüche mit einem Nenner kleiner oder gleich $M$ der Grösse nach, dann erhält man die Farey-Folge der Ordnung $M$. Aufgrund ihrer besonderen Eigenschaften sind die Farey-Folgen für Approximationsprobleme besonders gut geeignet.
} 
Satz 9 Es sei $f: \mathbb{N}_{0} \rightarrow \mathbb{N}$ eine streng monoton wachsende Funktion. Ferner sei $M \in \mathbb{R}$, $M \geq f(1)$ und $r \in \mathbb{R}$. Dann existieren zwei Zahlen $n_{1}, n_{2} \in \mathbb{N}_{0}$ und ein gekürzter Bruch $\frac{A}{B}$, mit $A \in \mathbb{Z}, 0<B=f\left(n_{1}\right)-f\left(n_{2}\right)<M$, so dass

$$
\left|r-\frac{A}{B}\right|<\frac{1}{g(M) B} \quad\left(\leq \frac{1}{g(B) B}, \quad \text { falls } B \geq f(0)\right) .
$$

Dabei ist $g(x):=\max \left\{y \in \mathbb{N}_{0} \mid f(y) \leq x\right\}$ für $x \geq f(0)$.

Beweis. Eine beliebige Zahl $r \in \mathbb{R}$ soll approximiert werden mit $\frac{A}{B}$, mit $A, B \in \mathbb{Z}$ und mit $0<B=f\left(n_{1}\right)-f\left(n_{2}\right)<M$ für geeignete $n_{1}, n_{2} \in \mathbb{N}_{0}$. Für $t_{1}, t_{2} \in \mathbb{N}_{0}$ mit $t_{1}<t_{2} \leq g(M)$ gilt $0<f\left(t_{2}\right)-f\left(t_{1}\right)<M$. Nun gilt: $z_{t}=f(t) r-[f(t) r] \in[0,1)$ für $t=0,1,2, \ldots, g(M)$. Von diesen $g(M)+1$ Zahlen liegen mindestens zwei im gleichen Teilintervall $\left[\frac{i-1}{g(M)}, \frac{i}{g(M)}\right)$ für $i=1,2, \ldots, g(M)$ (Schubfachprinzip). Also existieren $t_{1}$ und $t_{2} \in\{0,1,2, \ldots, g(M)\}$ mit $t_{1}<t_{2}$ und mit $\left|z_{t_{2}}-z_{t_{1}}\right|<\frac{1}{g(M)}$.

Sei nun $B=f\left(t_{2}\right)-f\left(t_{1}\right)$ und $A=\left[f\left(t_{2}\right) r\right]-\left[f\left(t_{1}\right) r\right]$, somit also $A \in \mathbb{Z}$ und $B \in \mathbb{N}$ mit $B<M$. Dann gilt:

$$
\begin{aligned}
\left|z_{t_{2}}-z_{t_{1}}\right| & =\left|\left(f\left(t_{2}\right) r-\left[f\left(t_{2}\right) r\right]\right)-\left(f\left(t_{1}\right) r-\left[f\left(t_{1}\right) r\right]\right)\right| \\
& =\left|\left(f\left(t_{2}\right)-f\left(t_{1}\right)\right) r-\left(\left[f\left(t_{2}\right) r\right]-\left[f\left(t_{1}\right) r\right]\right)\right| \\
& =|B r-A|<\frac{1}{g(M)},
\end{aligned}
$$

woraus unmittelbar folgt:

$$
\left|r-\frac{A}{B}\right|<\frac{1}{g(M) B} .
$$

Mit $B<M$, also $g(B) \leq g(M)$, folgt schliesslich auch die Klammerbemerkung.

Zu Satz 9 seien hier drei Beispiele angefügt.

Beispiel 1 Für $f(n)=n$ ist $g(M)=[M]$, und es ergibt sich wiederum unmittelbar DIRICHLETs Satz 6.

Beispiel 2 Ist $f(n)$ die $(n+1)$-te Primzahl, also $f(0)=2, f(1)=3, f(2)=5$ etc., dann ist $g(M)=\pi(M)-1$. Dabei bezeichnet $\pi(M)$ die Primzahlfunktion, sie bestimmt die Anzahl der Primzahlen $p_{i} \leq M$. Dies bedeutet: Für alle $r \in \mathbb{R}$ und für alle $M \geq 3$ existiert $A \in \mathbb{Z}$ und $B \in \mathbb{N}$ mit $B \leq M$ und $B=p_{1}-p_{2}$ (Differenz zweier Primzahlen), so dass gilt

$$
\left|r-\frac{A}{B}\right|<\frac{1}{(\pi(M)-1) B} \quad\left(\leq \frac{1}{(\pi(B)-1) B}\right) .
$$

Für $M>2$ gilt $\pi(M)>\frac{2 M}{3 \ln M}$ (MÖNKEMEYER [5]), womit also auch die folgende Abschätzung zutrifft:

Für alle $r \in \mathbb{R}$ und für alle $M>2$ existiert $A \in \mathbb{Z}$ und $B \in \mathbb{N}$ mit $B \leq M$ und $B=p_{1}-p_{2}$ (Differenz zweier Primzahlen), so dass gilt

$$
\left|r-\frac{A}{B}\right|<\frac{1}{\left(\frac{2 M}{3 \ln M}-1\right) B}=\frac{3 \ln M}{(2 M-3 \ln M) B} .
$$


Beispiel 3 Ist $f(n)=n^{p}$ (mit $p \in \mathbb{N}$ ), dann ist $g(M)=[\sqrt[p]{M}]$. Dies bedeutet: Für alle $r \in \mathbb{R}$, für alle $p \in \mathbb{N}$ und für alle $M \geq 1$ existiert $A \in \mathbb{Z}$ und $B \in \mathbb{N}$ mit $B \leq M$ und mit $B=n^{p}-m^{p}\left(n, m \in \mathbb{N}_{0}\right)$, so dass gilt:

$$
\left|r-\frac{A}{B}\right|<\frac{1}{[\sqrt[p]{M}]_{B}} \quad\left(\leq \frac{1}{[\sqrt[p]{B}] B}\right) .
$$

Im Folgenden werden zwei Approximationen untersucht, bei welchen die Bruchzahlen $\frac{A}{B}$ die Bedingung zu erfüllen haben, dass der Nenner $B$ als Produkt einer bestimmten Anzahl von natürlichen Faktoren $\beta_{i}$ dargestellt werden kann, wobei diese Faktoren aus einer beschränkten Teilmenge von $\mathbb{N}$ stammen müssen, also etwa $1<m \leq \beta_{i} \leq M$ für alle $i$.

Satz 10 Es sei $M \geq 1$ eine beliebige Schranke und $n \in \mathbb{N}$ eine natürliche Zahl. Dann existiert zu jedem $r \in \mathbb{R}$ mindestens eine rationale Zahl $\frac{A}{B}$, mit $B=\beta^{n}$ sowie $A=\alpha^{n}$, $(\alpha \in \mathbb{Z}$ und $\beta \in \mathbb{N}$ ) und mit $B \leq M$ (also $\beta \leq \sqrt[n]{M})$, so dass gilt

$$
\left|r-\frac{A}{B}\right|<\left(\sqrt[n]{|r|}+\frac{1}{\sqrt[n]{M B}}\right)^{n}-|r| \leq \frac{n}{\sqrt[n]{M B}}\left(\sqrt[n]{|r|}+\frac{1}{\sqrt[n]{M B}}\right)^{n-1} .
$$

Beweis. Grundsätzlich können wir uns auf $r \geq 0$ beschränken. Sei also $r \geq 0$ und $\sqrt[n]{r}$ eine reelle $n$-te Wurzel von $r$. Gemäss Satz 6 existiert mindestens ein $\frac{\alpha}{\beta}$ mit $\alpha \in \mathbb{Z}, \beta \in \mathbb{N}$ und mit $\beta \leq \sqrt[n]{M}$, so dass gilt

$$
\left|\sqrt[n]{r}-\frac{\alpha}{\beta}\right|<\frac{1}{\sqrt[n]{M} \beta}=: \Delta
$$

Damit ist

$$
\left|r-\left(\frac{\alpha}{\beta}\right)^{n}\right|<\max \left\{(\sqrt[n]{r}+\Delta)^{n}-r, r-(\sqrt[n]{r}-\Delta)^{n}\right\}=(\sqrt[n]{r}+\Delta)^{n}-r .
$$

Im Weiteren gilt für die auf $[0, \infty)$ konvexe Funktion $f(x)=x^{n}(n \in \mathbb{N})$

$$
(x+\Delta)^{n}-x^{n} \leq \Delta f^{\prime}(x+\Delta)=\Delta n(x+\Delta)^{n-1},
$$

weshalb weiter abgeschätzt werden kann:

$$
(\sqrt[n]{r}+\Delta)^{n}-r \leq \Delta n(\sqrt[n]{r}+\Delta)^{n-1} .
$$

Mit $\Delta=\frac{1}{\sqrt[n]{M} \beta}=\frac{1}{\sqrt[n]{M B}}$ folgt schliesslich Satz 10 .

Eine weitere Aussage in Bezug auf Approximationsbrüche mit zerlegbaren Zählern und Nennern macht der folgende

Satz 11 Zu jeder reellen Zahl $r \in \mathbb{R}$ und zu zwei beliebigen natürlichen Schranken $n, M \in$ $\mathbb{N}$ mit $2 \leq n \leq M$ existiert mindestens eine Zahl $\frac{A}{B}$ mit $A=A_{1} A_{2}, B=B_{1} B_{2}\left(A_{1}, A_{2} \in\right.$ $\left.\mathbb{Z}, B_{1}, B_{2} \in \mathbb{N}\right)$ mit $n \leq B_{i} \leq M$, so dass gilt:

$$
\left|r-\frac{A}{B}\right|<\frac{1+2 k M \sqrt{|r|}}{k^{2} B} .
$$

Dabei ist $k=\left[\frac{M}{n}\right]$. 
Bemerkung. Die Einschränkung auf $B_{1} \geq 2$ und $B_{2} \geq 2$ (und damit auf $B \geq 4$ ) ist sinnvoll, weil der Fall mit $B_{1}=1$ (resp. mit $B_{2}=1$ ) bereits mit Satz 6 wesentlich besser abgeschätzt werden kann.

Beweis. Grundsätzlich können wir uns wie beim Beweis des Satzes 10 auf $r \geq 0$ beschränken. Sei also $r \geq 0, \sqrt{r} \geq 0$ sowie $n, M \in \mathbb{N}$ mit $2 \leq n \leq M$ beliebig. Gemäss Satz 7 existiert mindestens eine Zahl $\frac{A}{B}$ mit $A \in \mathbb{Z}, B \in \mathbb{N}, n \leq B \leq M$, so dass gilt

$$
\left|\sqrt{r}-\frac{A}{B}\right|<\frac{1}{\left[\frac{M}{n}\right] B}=\frac{1}{k B}=: \Delta .
$$

Es seien nun $\frac{A_{1}}{B_{1}}$ und $\frac{A_{2}}{B_{2}}$ zwei solche (nicht notwendigerweise verschiedene) Zahlen. Nun denken wir uns die Zahl $r$ als den Flächeninhalt eines Quadrates der Seitenlänge $\sqrt{r}$ und $\frac{A_{1}}{B_{1}} \frac{A_{2}}{B_{2}}$ als den Flächeninhalt des Rechtecks mit den Seitenlängen $\frac{A_{1}}{B_{1}}$ und $\frac{A_{2}}{B_{2}}$.

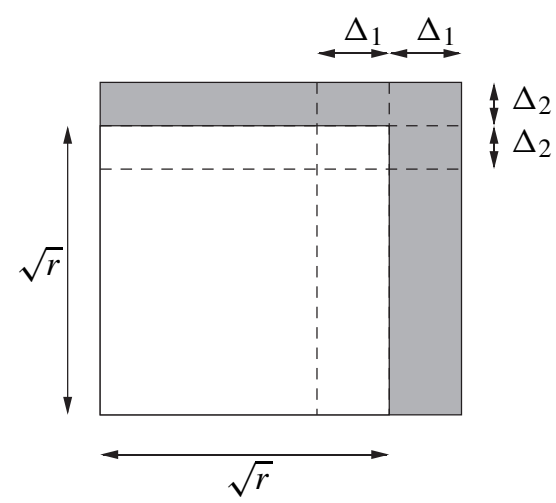

Die Seitenlängen der beiden Rechtecke unterscheiden sich gemäss (6) um weniger als $\Delta_{1}$ resp. $\Delta_{2}$. Für die Differenz der beiden Flächeninhalte gilt damit

$$
\begin{aligned}
\left|r-\frac{A_{1}}{B_{1}} \frac{A_{2}}{B_{2}}\right| & <\max \left\{\left(\sqrt{r}+\Delta_{1}\right)\left(\sqrt{r}+\Delta_{2}\right)-r, r-\left(\sqrt{r}-\Delta_{1}\right)\left(\sqrt{r}-\Delta_{2}\right)\right\} \\
& =\left(\sqrt{r}+\Delta_{1}\right)\left(\sqrt{r}+\Delta_{2}\right)-r
\end{aligned}
$$

da sowohl $\sqrt{r}$ als auch $\Delta_{i}$ positiv sind. Also gilt:

$$
\begin{aligned}
\left|r-\frac{A_{1}}{B_{1}} \frac{A_{2}}{B_{2}}\right| & <\Delta_{1} \Delta_{2}+\sqrt{r}\left(\Delta_{1}+\Delta_{2}\right) \\
& =\frac{1}{k^{2} B_{1} B_{2}}+\frac{\sqrt{r}}{k}\left(\frac{1}{B_{1}}+\frac{1}{B_{2}}\right) \\
& =\frac{1}{k^{2} B_{1} B_{2}}+\frac{\sqrt{r}}{k} \frac{B_{1}+B_{2}}{B_{1} B_{2}} \\
& =\frac{1+k \sqrt{r}\left(B_{1}+B_{2}\right)}{k^{2} B_{1} B_{2}} .
\end{aligned}
$$


Mit $B_{1}+B_{2} \leq 2 M$ sowie mit $A_{1} A_{2}=A$ und $B_{1} B_{2}=B$ folgt schliesslich:

$$
\left|r-\frac{A}{B}\right|<\frac{1+2 k M \sqrt{r}}{k^{2} B},
$$

wobei für $r<0$ im Abschätzungsterm $\sqrt{r}$ durch $\sqrt{|r|}$ ersetzt werden muss.

\section{Approximationsalgorithmen}

Dieser Abschnitt befasst sich mit der Suche nach möglichst guten Approximationen einer beliebigen reellen Zahl $r$ gemäss $\left|r-\frac{A}{B}\right|$, wenn die ganzen Zahlen $A$ und $B$ (mit $B>0$ ) aus bestimmten (endlichen) Teilmengen von $\mathbb{Z}$ (resp. von $\mathbb{N}$ ) stammen sollen.

Wir werden sehen, dass es sich bei diesen „Algorithmen“ eigentlich immer um die Bestimmung einer (möglichst kleinen) endlichen Menge von in Frage kommenden Bruchzahlen $\frac{A}{B}$ handelt, unter welchen anschliessend der beste Näherungsbruch lediglich durch ein möglichst kurzes „Versuchsverfahren“ ermittelt werden kann. Das klassische Beispiel dieser Ausgangslage bildet die Bestimmung von

$$
\min _{B \leq M}\left|r-\frac{A}{B}\right|,
$$

wo also der Nenner $B$ durch eine bestimmte obere (und in der Regel natürliche) Schranke $M$ begrenzt sein soll. Dabei wird meistens auf eine Begrenzung des Zählers $A$ verzichtet, was aber aufgrund der Tatsache, dass der Zähler $A$ ohnehin durch den Nenner $B$ determiniert wird, unserer allgemeinen Ausgangslage entspricht. Die Lösung dieses Problems scheint bereits CHRISTIAN HUYGENS bekannt gewesen zu sein, obwohl zu seiner Zeit ein Beweisverfahren vermutlich noch nicht bekannt war. Jedenfalls hat er davon Gebrauch gemacht bei der Konstruktion eines mechanischen Planetariums (HUYGENS [1]).

Heute bedient man sich dazu der umfangreichen Kenntnisse der Eigenschaften der Kettenbruchdarstellung reeller Zahlen. Es sei für die Herleitungen der folgenden Aussagen verwiesen auf die entsprechende Fachliteratur, beispielsweise auf das Standardwerk von O. PERRON [6].

Wir gehen hier aus von der Kettenbruchdarstellung einer beliebigen reellen Zahl $r$, also von der Darstellung $r=\left[q_{0} ; q_{1}, q_{2}, q_{3}, \ldots\right], q_{0} \in \mathbb{Z}, q_{i} \in \mathbb{N}$ für $i \geq 1$. Den Bruch $\frac{A_{i}}{B_{i}}=$ $\left[q_{0} ; q_{1}, q_{2}, \ldots, q_{i}\right]$ (für $i=0,1,2, \ldots$ ) bezeichnet PERRON als den $i$-ten Hauptnäherungsbruch der Zahl $r$, und wir wollen diese Bezeichnung hier ebenfalls verwenden. Falls $r$ rational ist, ist $i \leq n$, wobei für den letzten der $n+1$ Hauptnäherungsbrüche von $r$ dann gilt $\frac{A_{n}}{B_{n}}=r$. Die Hauptnäherungsbrüche weisen zahlreiche interessante Eigenschaften auf, von welchen wir hier vier festhalten:

E1 Die Hauptnäherungsbrüche einer Zahl $r$ sind stets beste Näherungen der Zahl $r$ in dem Sinne, dass jeder andere Bruch, welcher mindestens so nahe bei $r$ liegt, einen grösseren Nenner aufweist als der betrachtete Hauptnäherungsbruch. Für $i=0$ trifft diese Eigenschaft allerdings nur dann zu, wenn $q_{1}>1$ ist. 
E2 Die Hauptnäherungsbrüche approximieren den Wert $r$ alternierend, so dass gilt

$$
\left|r-\frac{A_{i+1}}{B_{i+1}}\right|<\left|r-\frac{A_{i}}{B_{i}}\right| \quad \text { für alle } i \geq 0
$$

und

$$
\frac{A_{0}}{B_{0}}<\frac{A_{2}}{B_{2}}<\ldots<r<\ldots<\frac{A_{3}}{B_{3}}<\frac{A_{1}}{B_{1}} .
$$

E3 Zähler $A_{i}$ und Nenner $B_{i}$ der Hauptnäherungsbrüche einer Zahl $r=\left[q_{0} ; q_{1}, q_{2}, q_{3}\right.$, ...] können direkt rekursiv bestimmt werden gemäss

$$
\begin{aligned}
& A_{i}=q_{i} A_{i-1}+A_{i-2}, \\
& B_{i}=q_{i} B_{i-1}+B_{i-2}
\end{aligned}
$$

für $i=1,2,3, \ldots$, mit den Startwerten $A_{-1}=1, A_{0}=q_{0}, B_{-1}=0, B_{0}=1$.

E4 Für auf diese Weise bestimmte Zähler und Nenner zweier aufeinander folgender Hauptnäherungsbrüche $\frac{A_{i}}{B_{i}}$ und $\frac{A_{i+1}}{B_{i+1}}$ gilt:

$$
B_{i} A_{i+1}-B_{i+1} A_{i}=(-1)^{i+1} .
$$

Damit sind insbesondere Zähler und Nenner eines jeden Hauptnäherungsbruches teilerfremd, die Hauptnäherungsbrüche sind also bereits vollständig gekürzt.

Allerdings vermögen die Hauptnäherungsbrüche einer reellen Zahl $r$ das Problem, den Wert $\min _{B \leq M}\left|r-\frac{A}{B}\right|$ resp. den bestmöglichen Näherungsbruch $\frac{A}{B}$ zu bestimmen, noch nicht vollständig zu lösen. Die dazu entscheidende Eigenschaft E1 besitzen nämlich noch weitere Brüche: Die Brüche $\frac{c A_{i-1}+A_{i-2}}{c B_{i-1}+B_{i-2}}$ (mit $c \in \mathbb{N}$ ) haben unter Umständen ebenfalls die Eigenschaft „,beste Näherungen“" von $r=\left[q_{0} ; q_{1}, q_{2}, q_{3}, \ldots\right]$ zu sein, und zwar genau dann, wenn gilt $\frac{q_{i}}{2}<c<q_{i}$, und zudem für $c=\frac{q_{i}}{2}$, falls $\left[q_{i} ; q_{i-1}, q_{i-2}, \ldots, q_{1}\right]>$ $\left[q_{i} ; q_{i+1}, q_{i+2}, \ldots\right]$. Bei diesen weiteren besten Näherungen spricht PERRON von Nebennäherungsbrüchen. Für die Nenner $c B_{i-1}+B_{i-2}$ dieser Nebennäherungsbrüche gilt gemäss der Eigenschaft E3: $B_{i-1}<c B_{i-1}+B_{i-2}<B_{i}$. Ihr Wert liegt somit zwischen den Nennern zweier benachbarter Hauptnäherungsbrüche.

Für die entsprechenden Herleitungen und insbesondere für den Beweis des folgenden Satzes orientiere man sich etwa bei O. PERRON [6].

Satz 12 Es sei $r=\left[q_{0} ; q_{1}, q_{2}, q_{3}, \ldots\right]$ eine beliebige reelle Zahl und $M \geq q_{2}+1 . \frac{Z_{1}}{N_{1}}$, $\frac{Z_{2}}{N_{2}}, \frac{Z_{3}}{N_{3}}, \ldots, \frac{Z_{k}}{N_{k}}$ sei die (endliche) Folge aller Haupt- und Nebennäherungsbrüche von $r$, mit $N_{i} \leq M$ (für alle $i=1,2, \ldots, k$ ), und zwar geordnet nach steigendem Nenner. Dann gilt:

$$
\min _{B \leq M}\left|r-\frac{A}{B}\right|=\left|r-\frac{Z_{k}}{N_{k}}\right| .
$$

Ist in der Kettenbruchdarstellung von $r=\left[q_{0} ; q_{1}, q_{2}, q_{3}, \ldots\right]$ der Teilnenner $q_{1}=1$, dann ist der Hauptnäherungsbruch $\frac{A_{0}}{B_{0}}=\frac{q_{0}}{1}$ in dieser Folge $\frac{Z_{i}}{N_{i}}$ wegzulassen. 
Beispiel. Es sei etwa $r=[6 ; 2,1,3,7,3]\left(=\frac{1^{\prime} 597}{251} \cong 6.3625498\right)$. Die Hauptnäherungsbrüche ( $*$ ) und die Nebennäherungsbrüche von $r$, deren Nenner kleiner sind als $M=100$, lauten in der Reihenfolge aufsteigender Nenner:
(*) $\frac{Z_{1}}{N_{1}}=\frac{6}{1}$
$\left|r-\frac{Z_{1}}{N_{1}}\right| \cong 0.36255$
(*) $\frac{Z_{2}}{N_{2}}=\frac{13}{2}$
$\left|r-\frac{Z_{2}}{N_{2}}\right| \cong 0.13745$
(*) $\frac{Z_{3}}{N_{3}}=\frac{19}{3}$
$\left|r-\frac{Z_{3}}{N_{3}}\right| \cong 0.02922$
$\frac{Z_{4}}{N_{4}}=\frac{51}{8}$
$\left|r-\frac{Z_{4}}{N_{4}}\right| \cong 0.01245$
(*) $\frac{Z_{5}}{N_{5}}=\frac{70}{11}$
$\left|r-\frac{Z_{5}}{N_{5}}\right| \cong 0.00109$
$\frac{Z_{6}}{N_{6}}=\frac{299}{47}$
$\left|r-\frac{Z_{6}}{N_{6}}\right| \cong 8.4710^{-4}$
$\frac{Z_{7}}{N_{7}}=\frac{369}{58}$
$\left|r-\frac{Z_{7}}{N_{7}}\right| \cong 4.8110^{-4}$
$\frac{Z_{8}}{N_{8}}=\frac{439}{69}$
$\left|r-\frac{Z_{8}}{N_{8}}\right| \cong 2.3110^{-4}$
(*) $\frac{Z_{9}}{N_{9}}=\frac{509}{80}$
$\left|r-\frac{Z_{9}}{N_{9}}\right| \cong 4.9810^{-5}$

Gemäss Satz 12 bildet also der Bruch $\frac{509}{80}$ die beste Approximation der Zahl $r=\frac{1 \text { '597 }}{251} \cong$ 6.3625498, wenn der Nenner den Wert $M=100$ nicht überschreiten darf.

Entsprechend wäre der Bruch $\frac{439}{69}$ die beste Approximation dieser Zahl $r$, wenn der Nenner etwa den Wert $M=75$ nicht überschreiten dürfte.

Dass in diesem Beispiel die Zahl $r$ rational ist, ist übrigens ohne Belang, und die Bestimmung der Folge $\frac{Z_{i}}{N_{i}}$ für irrationale Werte von $r$ ist nicht aufwändiger als für rationale Werte.

Satz 13 Es seien $\frac{A_{1}}{B_{1}}$ und $\frac{A_{2}}{B_{2}}$ zwei Bruchzahlen mit $\frac{A_{1}}{B_{1}}<\frac{A_{2}}{B_{2}}$, mit $A_{1}, A_{2} \in \mathbb{Z}, B_{1}, B_{2} \in \mathbb{N}$ sowie mit $A_{2} B_{1}-A_{1} B_{2}=1$. Ferner sei $\frac{p}{q}$ eine Bruchzahl im Intervall $\left[\frac{A_{1}}{B_{1}}, \frac{A_{2}}{B_{2}}\right]$ mit $p \in \mathbb{Z}$ und $q \in \mathbb{N}$. Dann existieren zwei Zahlen $t, s \in \mathbb{N}_{0}$, so dass gilt:

$$
\frac{p}{q}=\frac{t A_{1}+s A_{2}}{t B_{1}+s B_{2}}
$$

Beweis. Aus dem linearen Gleichungssystem

$$
\begin{aligned}
& p=t A_{1}+s A_{2} \\
& q=t B_{1}+s B_{2}
\end{aligned}
$$


folgt unmittelbar

$$
\begin{aligned}
s & =\frac{p B_{1}-q A_{1}}{A_{2} B_{1}-A_{1} B_{2}}, \\
t & =\frac{q A_{2}-p B_{2}}{A_{2} B_{1}-A_{1} B_{2}} .
\end{aligned}
$$

Der gemeinsame Nenner besitzt nach Voraussetzung den Wert 1. Wegen $\frac{A_{1}}{B_{1}} \leq \frac{p}{q} \leq \frac{A_{2}}{B_{2}}$ gilt für die ganzzahligen Zähler $p B_{1}-q A_{1} \geq 0$ und $q A_{2}-p B_{2} \geq 0$. Damit haben wir tatsächlich $s \in \mathbb{N}_{0}$ und $t \in \mathbb{N}_{0}$.

Bemerkungen. Den Bruch $\frac{p}{q}=\frac{t A_{1}+s A_{2}}{t B_{1}+s B_{2}}$ (mit $s, t \in \mathbb{N}_{0}$ ) bezeichnen wir als einen Medianwert der beiden Brüche $\frac{A_{1}}{B_{1}}$ und $\frac{A_{2}}{B_{2}}$.

Die Voraussetzung $A_{2} B_{1}-A_{1} B_{2}=1$ des Satzes 13 wird beispielsweise erfüllt von zwei konsekutiven Haupt- oder Nebennäherungsbrüchen einer Zahl $r$. Sie wird jedoch ebenfalls erfüllt von zwei konsekutiven Brüchen einer Farey-Folge. Die Hauptnäherungsbrüche bieten sich jedoch für das nachfolgend beschriebene Vorgehen besonders gut an, weil zwei aufeinander folgende die zu approximierende Zahl $r$ stets zwischen sich „einrahmen“.

\section{Vorgehen}

Es soll eine reelle Zahl $r$ approximiert werden mit einer Bruchzahl $\frac{A}{B}$, wobei der Nenner $B$ (und/oder der Zähler $A$ ) aus einer bestimmten (endlichen) Teilmenge $\mathbb{K}_{B} \subset \mathbb{N}$ (resp. $\mathbb{K}_{A} \subset$ $\mathbb{Z}$ ) stammen soll. Insbesondere gilt dann $B \leq M_{B}$ (und/oder $|A| \leq M_{A}$ ). Bestimmt man dann zwei konsekutive Hauptnäherungsbrüche der Zahl $r$, so ist gemäss der Eigenschaft E2 der eine kleiner und der andere grösser als die Zahl $r$, also etwa $\frac{A_{i}}{B_{i}}<r<\frac{A_{i+1}}{B_{i+1}}$. Jeder Näherungsbruch von $r$, welcher mindestens so nahe bei $r$ liegt wie $\frac{A_{i+1}}{B_{i+1}}$, muss nach Satz 13 ein Medianwert dieser beiden Brüche $\frac{A_{i}}{B_{i}}$ und $\frac{A_{i+1}}{B_{i+1}}$ sein, er muss somit die Form $\frac{A}{B}=\frac{t A_{i}+s A_{i+1}}{t B_{i}+s B_{i+1}}$ aufweisen (mit $t, s \in \mathbb{N}_{0}$ ). Da zudem Nenner (und/oder der Betrag des Zählers) lediglich eine beschränkte Grösse aufweisen dürfen, muss ferner gelten

$$
B(t, s):=t B_{i}+s B_{i+1} \leq M_{B} \text { und/oder }|A(t, s)|:=\left|t A_{i}+s A_{i+1}\right| \leq M_{A} .
$$

Damit wird die Menge der möglichen Bruchzahlen sehr eingeschränkt, und eine Prüfung danach, welche dieser Nenner (und/oder Zähler) Elemente der geforderten Teilmenge $\mathbb{K}_{B}$ (und/oder $\mathbb{K}_{A}$ ) sind, ist leicht möglich. Aus den verbleibenden Brüchen lässt sich danach der beste Approximationsbruch mit geringem Aufwand bestimmen. Praktischerweise werden diese letzten beiden Schritte mit einem geeigneten Computerprogramm durchgeführt.

Beispiel. Die Kreiszahl

$$
\pi=[3 ; 7,15,1,292,1,1,1,2,1,4, \ldots]
$$

soll möglichst gut approximiert werden mit einem Bruch $\frac{A}{B}$, wobei Zähler $A$ und Nenner $B$ natürlich und prim sein sollen, und wo $A$ und $B$ die Zahl $10^{\prime} 000$ nicht überschreiten 
dürfen. Die ersten fünf Hauptnäherungsbrüche von $\pi$ lauten:

$$
\frac{3}{1}, \frac{22}{7}, \frac{333}{106}, \frac{355}{113}, \frac{103^{\prime} 993}{33^{\prime} 102}, \ldots
$$

Weil Zähler (und Nenner) des letzten aufgeführten Hauptnäherungsbruches den Wert $10^{\prime} 000$ bereits übertreffen, wird man die Suche beginnen unter den Medianwerten der beiden Brüche $\frac{333}{106}$ und $\frac{355}{113}$. Jeder Approximationsbruch, welcher mindestens so nahe bei $\pi$ liegt wie der Bruch $\frac{355}{113}$, wird also in der Form $\frac{A}{B}=\frac{333 t+355 s}{106 t+113 s}$ (mit $t, s \in \mathbb{N}_{0}$ ) darstellbar sein müssen. Und da eine Beschränkung der Grösse von Zähler und Nenner mit dem Wert $10^{\prime} 000$ gegeben ist, müssen die beiden nicht-negativen und ganzzahligen Werte $t$ und $s$ die Bedingung erfüllen:

$$
333 t+355 s \leq 10^{\prime} 000 .
$$

Diese Bedingung wird von 393 nicht-negativen Zahlenpaaren $(t, s)$ erfüllt. In diesem Fall führt nur ein einziges dieser 393 Zahlenpaare zu zwei Primzahlen:

$$
\begin{aligned}
& 333 \cdot 4+355 \cdot 23=9^{\prime} 497 \in \mathbb{P}, \\
& 106 \cdot 4+113 \cdot 23=3^{\prime} 023 \in \mathbb{P} .
\end{aligned}
$$

Damit wird unter den gegebenen Bedingungen der Bruch $\frac{9^{\prime} 497}{3^{\prime} 023}$ mit Sicherheit die bestmögliche Approximation der Zahl $\pi$ sein. Falls man unter den Medianwerten der beiden Hauptnäherungsbrüche $\frac{333}{106}$ und $\frac{355}{113}$ keinen Bruch hätte finden können, welcher die verlangten Bedingungen zu erfüllen vermag, dann hätte man die Suche auf dieselbe Weise weiterführen müssen unter den Medianwerten der beiden konsekutiven Hauptnäherungsbrüche mit den nächst kleineren Nennern, also mit $\frac{22}{7}$ und $\frac{333}{106}$.

Zur Veranschaulichung seien hier einige weitere Beispiele von optimalen Approximatio-

\begin{tabular}{|c|c|c|c|}
\hline $\begin{array}{l}\text { Schranke } \rightarrow \\
\text { Bedingung } \downarrow\end{array}$ & $\leq 100$ & $\leq 1^{\prime} 000$ & $\leq 10^{\prime} 000$ \\
\hline $\begin{array}{l}\text { keine zusätzlichen } \\
\text { Bedingungen }\end{array}$ & $\begin{array}{l}\frac{22}{7} \\
\Delta \cong 1.2645 \cdot 10^{-3}\end{array}$ & $\begin{array}{l}\frac{355}{113} \\
\Delta \cong 2.6676 \cdot 10^{-7}\end{array}$ & $\begin{array}{l}\frac{355}{113} \\
\Delta \cong 2.6676 \cdot 10^{-7}\end{array}$ \\
\hline $\begin{array}{l}\text { Zähler und } \\
\text { Nenner prim }\end{array}$ & $\begin{array}{l}\frac{97}{31} \\
\Delta \cong 1.2560 \cdot 10^{-2}\end{array}$ & $\begin{array}{l}\frac{619}{197} \\
\Delta \cong 5.3933 \cdot 10^{-4}\end{array}$ & $\begin{array}{l}\frac{9^{\prime} 497}{3^{\prime} 023} \\
\Delta \cong 1.1443 \cdot 10^{-5}\end{array}$ \\
\hline $\begin{array}{l}\text { alle Prim- } \\
\text { faktoren }>5\end{array}$ & $\begin{array}{l}\frac{91}{29} \\
\Delta \cong 3.6616 \cdot 10^{-3}\end{array}$ & $\frac{619}{197} \cong 5.3933 \cdot 10^{-4}$ & $\begin{array}{l}\frac{9^{\prime} 541}{3^{\prime} 037} \\
\Delta \cong 5.5611 \cdot 10^{-6}\end{array}$ \\
\hline $\begin{array}{l}\text { Zähler und } \\
\text { Nenner ungerade }\end{array}$ & $\begin{array}{l}\frac{91}{29} \\
\Delta \cong 3.6616 \cdot 10^{-3}\end{array}$ & $\begin{array}{l}\frac{355}{113} \\
\Delta \cong 2.6676 \cdot 10^{-7}\end{array}$ & $\begin{array}{l}\frac{355}{113} \\
\Delta \cong 2.6676 \cdot 10^{-7}\end{array}$ \\
\hline $\begin{array}{l}\text { je } 2 \text { Faktoren } \\
\text { beschränkter Grösse }\end{array}$ & $\begin{array}{l}\frac{51 \cdot 77}{25 \cdot 50} \\
\Delta \cong 7.3464 \cdot 10^{-6}\end{array}$ & $\begin{array}{l}\frac{359 \cdot 871}{298 \cdot 334} \\
\Delta \cong 2.8937 \cdot 10^{-11}\end{array}$ & \\
\hline $\begin{array}{l}\text { je } 3 \text { Faktoren } \\
\text { beschränkter Grösse }\end{array}$ & $\begin{array}{l}\frac{33 \cdot 47 \cdot 73}{17 \cdot 40 \cdot 53} \\
\Delta \cong 2.1216 \cdot 10^{-8}\end{array}$ & & \\
\hline
\end{tabular}
nen der Kreiszahl $\pi$ angeführt mit unterschiedlichen Bedingungen für Zähler und Nenner des Approximationsbruches. Mit $\Delta$ ist dabei jeweils auch der Approximationsfehler, also die Grösse $\left|\pi-\frac{A}{B}\right|$, angegeben. 


\begin{tabular}{|c|c|c|c|}
\hline $\begin{array}{l}\text { Schranke } \rightarrow \\
\text { Bedingung } \downarrow\end{array}$ & $\leq 100^{\prime} 000$ & $\leq 1^{\prime} 000^{\prime} 000$ & $\leq 10^{\prime} 000^{\prime} 000$ \\
\hline $\begin{array}{l}\text { keine zusätzlichen } \\
\text { Bedingungen }\end{array}$ & $\begin{array}{l}\frac{99^{\prime} 733}{31^{\prime} 746} \\
\Delta \cong 1.1997 \cdot 10^{-8}\end{array}$ & $\begin{array}{l}\frac{833^{\prime} 719}{265^{\prime} 381} \\
\Delta \cong 8.9233 \cdot 10^{-12}\end{array}$ & $\begin{array}{l}\frac{6^{\prime} 565^{\prime} 759}{2^{\prime} 089^{\prime} 946} \\
\Delta \cong 1 \cdot 10^{-13}\end{array}$ \\
\hline $\begin{array}{l}\text { Zähler und } \\
\text { Nenner prim }\end{array}$ & $\begin{array}{l}\frac{60^{\prime} 661}{19^{\prime} 309} \\
\Delta \cong 6.4986 \cdot 10^{-7}\end{array}$ & $\begin{array}{l}\frac{833^{\prime} 719}{265^{\prime} 381} \\
\Delta \cong 8.9233 \cdot 10^{-12}\end{array}$ & $\begin{array}{l}\frac{833^{\prime} 719}{265^{\prime} 381} \\
\Delta \cong 8.9233 \cdot 10^{-12}\end{array}$ \\
\hline $\begin{array}{l}\text { alle Prim- } \\
\text { faktoren }>5\end{array}$ & $\begin{array}{l}\frac{99^{\prime} 001}{31^{\prime} 513} \\
\Delta \cong 2.9488 \cdot 10^{-7}\end{array}$ & $\begin{array}{l}\frac{833^{\prime} 719}{265^{\prime} 381} \\
\Delta \cong 8.9233 \cdot 10^{-12}\end{array}$ & $\begin{array}{l}7^{\prime} 712^{\prime} 167 \\
2^{\prime} 454^{\prime} 859 \\
\Delta \cong 2.8766 \cdot 10^{-13}\end{array}$ \\
\hline $\begin{array}{l}\text { Zähler und } \\
\text { Nenner ungerade }\end{array}$ & $\begin{array}{l}\frac{99^{\prime} 711}{31^{\prime} 739} \\
\Delta \cong 2.9088 \cdot 10^{-7}\end{array}$ & $\begin{array}{l}\frac{833^{\prime} 719}{265^{\prime} 381} \\
\Delta \cong 8.9223 \cdot 10^{-12}\end{array}$ & $\begin{array}{l}\frac{5}{}^{\prime} 419^{\prime} 351 \\
\Delta \cong 1.8463 \cdot 10^{-13}\end{array}$ \\
\hline
\end{tabular}

\section{Grenzen der Approximierbarkeit}

Unter gewissen Umständen lässt sich die minimale Fehlergrösse bei der Diophantischen Approximation einer reellen Zahl $r$ abschätzen, also in der Weise

$$
\left|r-\frac{A}{B}\right| \geq c \quad \text { für alle } A, B \in \mathbb{G} \subset \mathbb{Z} .
$$

Diese Abschätzung kann einerseits durch die in Frage kommenden ganzen Zahlen $A$ und $B$ begründet sein, andererseits aber auch durch die Natur der zu approximierenden Zahl $r$ selbst. In diese Richtung führt beispielsweise ein Satz von J. LiOUVILLE, welcher besagt, dass eine algebraische Zahl der Ordnung $n$ höchstens mit der Ordnung $n$ approximiert werden kann, dass somit für jede algebraische Zahl $r n$-ter Ordnung eine positive Schranke $\lambda>0$ existiert, so dass für jeden rationalen Bruch $\frac{A}{B}$ gilt

$$
\left|r-\frac{A}{B}\right| \geq \frac{\lambda}{B^{n}} .
$$

Noch etwas weiter führt hier ein Satz von K.F. ROTH aus dem Jahre 1955, welcher nachweist, dass jede algebraische Irrationalzahl höchstens (und damit genau) mit der Ordnung 2 approximierbar ist.

Für den folgenden Satz sei $r=\left[q_{0} ; q_{1}, q_{2}, q_{3}, \ldots\right]$ wiederum die Kettenbruchdarstellung einer reellen Zahl $r$, und ferner seien die beiden reellen Zahlen $\alpha_{n}$ und $z_{n}$ definiert wie folgt:

$$
\begin{aligned}
\alpha_{n} & =\left[q_{n} ; q_{n-1}, q_{n-2}, \ldots, q_{1}\right] & & \text { für } n \geq 1, \\
z_{n} & =\left[q_{n} ; q_{n+1}, q_{n+2}, \ldots\right] & & \text { für } n \geq 0 .
\end{aligned}
$$

Damit ist $r=\left[q_{0} ; q_{1}, q_{2}, \ldots, q_{n-1}, z_{n}\right]$, und es gilt $\alpha_{n}=\frac{B_{n}}{B_{n-1}}$, wo $B_{n}$ und $B_{n-1}$ die Nenner des $n$-ten bzw. des $(n-1)$-ten Hauptnäherungsbruches $\frac{A_{n}}{B_{n}}$ bzw. $\frac{A_{n-1}}{B_{n-1}}$ der zu approximierenden Zahl $r$ darstellen. Schliesslich gilt:

$$
z_{0}=r=\frac{z_{n+1} A_{n}+A_{n-1}}{z_{n+1} B_{n}+B_{n-1}} .
$$

Zur Herleitung dieser Zusammenhänge kann man sich beispielsweise orientieren bei PERRON [6, S. 27ff]. Damit stehen die Begriffe bereit zur Formulierung des folgenden Satzes: 
Satz 14 Es seien $\frac{A_{n-1}}{B_{n-1}}$ und $\frac{A_{n}}{B_{n}}$ (mit $n \geq 1$ ) zwei konsekutive Hauptnäherungsbrüche einer reellen Zahl $r$, und es seien $t$ und s zwei nicht-negative reelle Zahlen, wobei nicht beide gleich Null sein sollen. Dann gilt für $n \geq 1$

$$
\left|r-\frac{t A_{n}+s A_{n-1}}{t B_{n}+s B_{n-1}}\right|=\frac{1}{\lambda_{n}\left(t B_{n}+s B_{n-1}\right)^{2}}
$$

mit

$$
\lambda_{n}=\frac{z_{n+1} \alpha_{n}+1}{\left|z_{n+1} s-t\right|\left(t \alpha_{n}+s\right)} .
$$

Auf den Beweis von Satz 14 soll hier verzichtet werden. Zwei Sonderfälle, welche sich aus diesem Satz ergeben, sollen allerdings hervorgehoben werden:

$$
\begin{aligned}
& t=1, s=0:\left|r-\frac{A_{n}}{B_{n}}\right|=\frac{1}{\lambda_{n} B_{n}^{2}} \quad \text { mit } \lambda_{n}=z_{n+1}+\frac{1}{\alpha_{n}} \\
& t=1, s=1: \quad\left|r-\frac{A_{n}+A_{n-1}}{B_{n}+B_{n-1}}\right|=\frac{1}{\lambda_{n}\left(B_{n}+B_{n-1}\right)^{2}} \\
& \text { mit } \lambda_{n}=1+\frac{1}{z_{n+1}-1}-\frac{1}{\alpha_{n}+1} .
\end{aligned}
$$

Satz 14 kann herangezogen werden, um ein Mass für die Approximierbarkeit einer reellen Zahl $r$ nach unten abzuschätzen: Eine reelle Zahl $r$ sei zu approximieren mit Bruchzahlen $\frac{A}{B}(A \in \mathbb{Z}, B \in \mathbb{N})$ und mit $0<B \leq M$, wo $M$ eine gegebene Schranke darstelle. Wir gehen von den ersten beiden Hauptnäherungsbrüchen $\frac{A_{0}}{B_{0}}$ und $\frac{A_{1}}{B_{1}}$ der Zahl $r$ aus. Mit der Kettenbruchdarstellung $r=\left[q_{0} ; q_{1}, q_{2}, q_{3}, \ldots\right]$ gilt $A_{0}=q_{0}, B_{0}=1, A_{1}=q_{0} q_{1}+1$ und $B_{1}=q_{1}$. Ferner gilt $\frac{A_{0}}{B_{0}}<r<\frac{A_{1}}{B_{1}}$, und jede rationale Approximation der Zahl $r$, welche mindestens so nahe bei $r$ liegt wie der Hauptnäherungsbruch $\frac{A_{1}}{B_{1}}$, ist nach Satz 14 darstellbar in der Form

$$
\frac{A}{B}=\frac{t A_{1}+s A_{0}}{t B_{1}+s B_{0}}=\frac{t A_{1}+s A_{0}}{t q_{1}+s} \quad \text { mit } t, s \in \mathbb{N}_{0} .
$$

Sofern die gegebene Schranke $M$ die Bedingung $M \geq B_{1}=q_{1}$ erfüllt, wird mindestens der Hauptnäherungsbruch $\frac{A_{1}}{B_{1}}$ die geforderte Bedingung erfüllen, und jeder bessere Näherungsbruch wird in der Form (7) darstellbar sein müssen. Nach Satz 14 gilt für $n=1$

$$
\left|r-\frac{A}{B}\right|=\left|r-\frac{t A_{1}+s A_{0}}{t B_{1}+s B_{0}}\right|=\frac{1}{\lambda_{1} B^{2}}
$$

mit

$$
\lambda_{1}=\frac{z_{2} \alpha_{1}+1}{\left|z_{2} s-t\right|\left(t \alpha_{1}+s\right)}=\frac{z_{2} q_{1}+1}{\left|z_{2} s-t\right| B} .
$$

Dabei ist $\alpha_{1}=q_{1}$, und wegen $r=\left[q_{0} ; q_{1}, z_{2}\right]=q_{0}+\frac{1}{q_{1}+\frac{1}{z_{2}}}$ ergibt sich $z_{2}=\frac{r-q_{0}}{q_{0} q_{1}+1-q_{1} r}$. Mit der Abschätzung $r-q_{0}=\frac{1}{q_{1}+\frac{1}{\ldots}}<\frac{1}{q_{1}}$ gilt also $z_{2}<\frac{1}{q_{1}\left(q_{0} q_{1}+1-q_{1} r\right)}$, und damit ist

$$
\lambda_{1}<\frac{\frac{1}{q_{0} q_{1}+1-q_{1} r}+1}{\left|z_{2} s-t\right| B}=\frac{q_{0} q_{1}-q_{1} r+2}{\left|z_{2} s-t\right| B\left(q_{0} q_{1}-q_{1} r+1\right)} .
$$


Nun bleibt noch, den Betrag $\Delta:=\left|z_{2} s-t\right|$ nach oben abzuschätzen. Dabei handelt es sich um eine „Approximation der zweiten Art“ für die reelle Zahl $z_{2}$ mit $s \in \mathbb{N}$ und $t \in \mathbb{Z}$. Da jedoch $z_{2}=\left[q_{2} ; q_{3}, q_{4}, \ldots\right]>q_{2} \geq 1$ ist, kann ohne Einschränkung der Allgemeinheit angenommen werden, dass nebst der Zahl $s$ auch die Zahl $t$ positiv sei. Bei den „Approximationen der zweiten Art" bilden die Hauptnäherungsbrüche die einzigen besten Näherungen (siehe z.B. PERRON [6]). Ferner gilt nach Voraussetzung $t A_{1}+s A_{0}=$ $t\left(q_{0} q_{1}+1\right)+s q_{0} \leq M$ sowie $t B_{1}+s B_{0}=t q_{1}+s \leq M$. Also ist insbesondere

$$
s \leq \frac{M-t\left(q_{0} q_{1}+1\right)}{q_{0}} \leq \frac{M-q_{0} q_{1}-1}{q_{0}} .
$$

Damit ist der Betrag $\Delta$ mindestens so gross, wie der Approximationsfehler $\left|z_{2} s-t\right|$, wenn $\frac{t}{s}$ der letzte Hauptnäherungsbruch der Zahl $z_{2}$ ist, für welchen diese Bedingung (8) erfüllt ist. Wenn wir also diesen Hauptnäherungsbruch von $z_{2}$ etwa mit $\frac{T(M)}{S(M)}$ bezeichnen, so ist $\Delta \geq\left|z_{2} S(M)-T(M)\right|$, und es ergibt sich

$$
\lambda_{1}<\frac{q_{0} q_{1}-q_{1} r+2}{\left|z_{2} S(M)-T(M)\right|\left(q_{0} q_{1}-q_{1} r+1\right) B} .
$$

Damit kann schliesslich die folgende Abschätzung nach unten formuliert werden:

Satz 15 Eine beliebige reelle Zahl $r=\left[q_{0} ; q_{1}, q_{2}, q_{3}, \ldots\right]$ soll approximiert werden mit einer rationalen Zahl $\frac{A}{B}(A \in \mathbb{Z}$ und $B \in \mathbb{N})$ mit $B \leq M$, wo $M \geq q_{0}\left(q_{1}+1\right)+1$ eine gegebene Schranke darstelle. Dann gilt für den Approximationsfehler

$$
\left|r-\frac{A}{B}\right|>\frac{\left|z_{2} S(M)-T(M)\right|\left(q_{0} q_{1}-q_{1} r+1\right)}{\left(q_{0} q_{1}-q_{1} r+2\right) B} .
$$

Dabei bezeichnen die beiden natürlichen Zahlen $T(M)$ und $S(M)$ Zähler und Nenner des letzten Hauptnäherungsbruches $\frac{t}{s}$ der Zahl $z_{2}$ mit $s \leq \frac{M-q_{0} q_{1}-1}{q_{0}}$.

\section{References}

[1] Huygens, Ch. (Hugenius): Opuscula posthuma. Descriptio automati planetarii. Lugduni Batavorum 1703.

[2] Khintchine, A.: Kettenbrüche. Teubner, Leipzig 1956.

[3] Lejeune Dirichlet, G.P.: Verallgemeinerung eines Satzes aus der Lehre von Kettenbrüchen nebst einigen Anwendungen auf die Theorie der Zahlen. S.-B. Preuss. Akad. Wiss. (1842), 93-95.

[4] Minkowski, H.: Diophantische Approximationen. Teubner, Leipzig 1927.

[5] Mönkemeyer, R.: Einführung in die Zahlentheorie. Hermann Schroedel, 1971.

[6] Perron, O.: Die Lehre von den Kettenbrüchen. Teubner, Stuttgart 1954.

[7] Robinson, R.M.: The approximation of irrational numbers by fractions with odd or even terms. Duke Math. J. 7, 1940 .

[8] Scott, W.T.: Approximation to real irrationals by certain classes of rational fractions. Bull. Amer. Math. Soc. 46 (1940), 124-129.

[9] Sierpinski, W.: Sur un théorème de la théorie des approximations rationelles. C. R. Soc. Sci. 2, 1909.

Robert Baggenstos

CH-4532 Feldbrunnen, Schweiz

e-mail: baggenstos@bluewin.ch 